Y. Sato

Nagoya Math. J.

Vol. 123 (1991), 119-139

\title{
DISTRIBUTIONS OF STABLE RANDOM FIELDS OF CHENTSOV TYPE
}

\author{
YUMIKO SATO
}

\section{§1. Introduction}

In this paper we discuss the determinism of distributions of some stable random fields which are constructed through integral-geometric method. The determinism depends on the dimension of the parameter space $R^{d}$.

We say that a family of random variables $\left\{X(t) ; t \in \mathbf{R}^{d}\right\}$ is a symmetric $\alpha$-stable (abbreviated to $S \alpha S$ ) random field on $\mathbf{R}^{d}$ if every finite linear combination $\sum_{i=1}^{n} a_{i} X\left(t_{i}\right)$ has a symmetric stable distribution of index $\alpha$. Let $(E, \mathscr{B}, \mu)$ be a measure space. We say that a family of random variables $\{Y(B) ; B \in \mathscr{B}, \mu(B)<\infty\}$ is the $S \alpha S$ random measure corresponding to $(E, \mathscr{B}, \mu)$ if (i) $E(\exp [i z Y(B)])=\exp \left(-\mu(B)|z|^{\alpha}\right)$, for $z \in \mathbf{R}$ and $\mu(B)<\infty$, (ii) $Y\left(B_{1}\right), Y\left(B_{2}\right), \cdots$ are independent whenever $B_{1}, B_{2}, \cdots$ are disjoint and $\mu\left(B_{j}\right)<\infty, j=1,2, \cdots$, (iii) $Y\left(\bigcup_{j=1}^{\infty} B_{j}\right)=\sum_{j=1}^{\infty} Y\left(B_{j}\right)$ a.s. whenever $B_{1}, B_{2}$, $\cdots$ are disjoint and $\mu\left(\bigcup_{j=1}^{\infty} B_{j}\right)<\infty$.

We define a class of $S \alpha S$ random fields with a particular choice of $E$. Let $E_{0}$ be the set of all $(d-1)$-dimensional spheres in $\mathbf{R}^{d}$. Any element of $E_{0}$ is expressed by a coordinate system $(r, x)$, where $(r, x)$ corresponds to the sphere with radius $r \in \mathbf{R}_{+}=(0, \infty)$ and center $x \in \mathbf{R}^{d}$. Thus we make the identification

$$
E_{0}=\left\{(r, x) ; r \in \mathbf{R}_{+}, x \in \mathbf{R}^{i}\right\} .
$$

For $t \in \mathbf{R}^{a}$, let $s_{t}$ be the set of all spheres which separate the point $t$ and the origin $O$, namely

$$
S_{t}=\{(r, x) ; d(x, O) \leqq r\} \triangle\{(r, x) ; d(r, x) \leqq r\},
$$

where $A \triangle B$ denotes the symmetric difference of $A$ and $B$ and $d(a, b)$ denotes the Euclidean distance between $a$ and $b$. Let $\mathscr{B}_{0}$ be the $\sigma$-algebra

Received October 23, 1990. 
of Borel sets in $E_{0}$. Given a measure $\mu$ on $\left(E_{0}, \mathscr{B}_{0}\right)$ such that

$$
\mu\left(S_{t}\right)<\infty \text { for all } t \in \mathbf{R}^{d},
$$

we define an $S \alpha S$ random field by

$$
X(t)=Y\left(S_{t}\right), \quad t \in \mathbf{R}^{d},
$$

using the $S \alpha S$ random measure $\{Y(B)\}$ corresponding to $\left(E_{0}, \mathscr{B}_{0}, \mu\right)$. We call this $\{X(t)\} S \alpha S$ random field of Chentsov type on $\mathbf{R}^{d}$ associated with $\mu$.

Such a random field is viewed as an extension of N.N. Chentsov's representation $Y\left(S_{t}^{\prime}\right)$ of Lévy's Brownian motion of $\mathbf{R}^{d}$-parameter. The $Y\left(S_{t}^{\prime}\right)$ is defined by Chentsov through Gaussian random measure $Y$ associated with a measure on the space $E^{\prime}$ of all hyperplanes of co-dimension 1 in $\mathbf{R}^{d}$ and the defining set $S_{t}^{\prime}$ is the set of all hyperplanes which separate $t$ and the origin $O$, [1], [3]. S. Takenaka, [7], applied this idea to stable case. Using $E_{0}$ in place of $E^{\prime}$, he proves that if $d \mu_{\beta}(r, x)=r^{\beta-d-1} d r d x$, $0<\beta<1$, then the Chentsov type $S \alpha S$ random field $X_{\alpha, \beta}(t)$ associated with $\left(E_{0}, \mu_{\beta}\right)$ is self-similar with exponent $H=\beta / \alpha$. For $d=1,\left\{X_{\alpha, \beta}(t)\right\}$ presents a new example of $S \alpha S, H$-self-similar process with stationary increments in the area of $\alpha$ and $H$ where no examples were known before.

The distributions of a Chentsov type $S \alpha S$ random field on $\mathbf{R}^{d}$ have a characteristic property which depends on the dimension $d$ of the parameters. We do not assume any condition other than (1.3) for the associated measure. The aim of this paper is to prove the following theorem.

Theorem 1. Let $0<\alpha<2$. Let $\mu$ be a measure on $\left(E_{0}, \mathscr{B}_{0}\right)$ satisfying (1.3) and let $\left\{X(t) ; t \in \mathbf{R}^{d}\right\}$ be the $S \alpha S$ random field of Chentsov type on $\mathbf{R}^{d}$ associated with $\mu$. Then, for any $n>d+1$ and for any distinct $t_{1}, \cdots, t_{n}$ $\in \mathbf{R}^{d}$, the distribution $\left(X\left(t_{1}\right), \cdots, X\left(t_{n}\right)\right)$ is determined by its $(d+1)$-dimensional marginal distributions.

CoROllary. Let $0<\alpha<2$. Let $\mu$ and $\tilde{\mu}$ be measures on $\left(E_{0}, \mathscr{B}_{0}\right)$ satisfying (1.3). Let $\left\{X(t) ; t \in \mathbf{R}^{d}\right\}$ and $\left\{\tilde{X}(t) ; t \in \mathbf{R}^{d}\right\}$ be the $S \alpha S$ random fields of Chentsov type associated with $\mu$ and $\tilde{\mu}$, respectively. If the $(d+1)$ dimensional distributions of $\{X(t)\}$ and $\{\tilde{X}(t)\}$ coincide, then $\{X(t)\}$ and $\{\tilde{X}(t)\}$ are equivalent, that is, the finite-dimensional distributions of $\{X(t)\}$ and $\{\tilde{X}(t)\}$ coincide.

Remark 1. The number $d+1$ in Theorem 1 is best possible in the following sense. There are two Chentsov type random fields $\{X(t)\}$ and 
$\{\tilde{X}(t)\}$ associated with $\mu$ and $\tilde{\mu}$, respectively, such that, for some $T=$ $\left(t_{1}, \cdots, t_{d+1}\right)$, the $d$-dimensional marginal distributions of $\left(X\left(t_{1}\right), \cdots, X\left(t_{d+1}\right)\right)$ and $\left(\tilde{X}\left(t_{1}\right), \cdots, \tilde{X}\left(t_{d+1}\right)\right)$ coincide but their $(d+1)$-dimensional distributions are different. (see Example 4.2)

Remark 2. If we take $E^{\prime}$ and $S_{t}^{\prime}$ instead of $E_{0}$ and $S_{t}$ and define

$$
X^{\prime}(t)=Y\left(S_{t}^{\prime}\right) \quad \text { for } t \in \mathbf{R}^{d},
$$

where $Y$ is an $S \alpha S$ random measure with $0<\alpha<2$ associated with a measure $\mu^{\prime}$ on $E^{\prime}$ satisfying $\mu^{\prime}\left(S_{t}^{\prime}\right),<\infty$, then we have determinism by $d$-dimensional marginal distributions instead of determinism by $(d+1)$ dimensional marginal distributions in Theorem 1. Namely, for any $n>d$ and any distinct $t_{1}, \cdots, t_{n} \in \mathbf{R}^{d}$, the distribution of $\left(X^{\prime}\left(t_{1}\right), \cdots, X^{\prime}\left(t_{n}\right)\right)$ is determined by its $d$-dimensional marginal distributions. This fact can be proved by a similar method as Theorem 1 .

Theorem 1 will be reduced to a geometric theorem concerning an intersection property of a family of cones in $\mathbf{R}_{+} \times \mathbf{R}^{d}$. The proof of this geometric theorem is an essential part of our argument. For $t \in \mathbf{R}^{d}$, set

$$
C_{t}=\{(r, x) ; d(x, t) \leqq r\} .
$$

Then, $C_{t}$ is a right cone in $\mathbf{R}_{+} \times \mathbf{R}^{d}$ with vertex $(0, t)$. Note that the point $(0, t)$ is not included in the space $\mathbf{R}_{+} \times \mathbf{R}^{d}$. Hereafter we simply call $C_{t}$ the cone with vertex $t$. The relation

$$
S_{t}=C_{0} \triangle C_{t}
$$

shows that, instead of $S_{t}$ 's, we may study $C_{t}$ 's. Given $m$ cones $C_{t_{1}}, \ldots, C_{t_{m}}$, we consider the partition of the set $\bigcup_{i=1}^{m} C_{t_{i}}$ generated by $\left\{C_{t_{i}}, i=1, \cdots, m\right\}$. Now set

$$
\mathscr{E}_{m}=\left\{e=\left(e_{1}, \cdots, e_{m}\right) ; e_{i}=0 \text { or } 1 \text { for } i=1, \cdots, m\right\} \backslash\{(0, \cdots, 0)\} .
$$

We call $e \in \mathscr{E}_{m}$ a label of size $m$ and $\mathscr{E}_{m}$ the label set. With the notation

$$
C_{t}^{1}=C_{t} \quad \text { and } \quad C_{t}^{0}=C_{t}^{e}=\left(\mathbf{R}_{+} \times \mathbf{R}^{d}\right) \backslash C_{t},
$$

we define

$$
C(T, e)=\bigcap_{i=1}^{m} C_{t_{i}}^{e_{i}}
$$

for $T=\left(t_{1}, \cdots, t_{m}\right) \in\left(\mathbf{R}^{d}\right)^{m}$ and $e=\left(e_{1}, \cdots, e_{m}\right) \in \mathscr{E}_{m}$. Then $C(T, e), e \in \mathscr{E}_{m}$, are disjoint sets and 


$$
\bigcup_{i=1}^{m} C_{t_{i}}=\bigcup_{e \in \varepsilon_{m}} C(T, e) \text {. }
$$

For $e=\left(e_{1}, \cdots, e_{m}\right) \in \mathscr{E}_{m}$, the complementary label $e^{*}$ of $e$ is defined by

$$
e^{*}=\left(e_{1}^{*}, \cdots, e_{m}^{*}\right), e_{i}+e_{i}^{*}=1 \text { for any } i .
$$

Theorem 2. If $m \geqq d+3$, then, for any $T=\left(t_{1}, \cdots, t_{m}\right) \in\left(\mathbf{R}^{d}\right)^{m}$, there exists a label $e \in \mathscr{E}_{m}$ such that $C(T, e)=\varnothing$ and $C\left(T, e^{*}\right)=\varnothing$.

In $\S 2$ we reduce Theorem 1 to Theorem 2 , which is proved in $\S 3$ after a series of lemmas. Concluding remarks are given in $\S 4$. The particular cases $d=1$ and 2 of Theorem 1 have been treated in the author's paper [4], [5]. Determinism under different defining sets in one-dimensional case will be discussed in a joint paper with S. Takenaka [6].

\section{§ 2. Reduction of Theorem 1 to Theorem 2}

Let $0<\alpha<2$. Let $\mu$ be a measure on $\left(E_{0}, \mathscr{B}_{0}\right)$ satisfying (1.3) and let $\left\{X(t) ; t \in \mathbf{R}^{d}\right\}$ be the $S \alpha S$ random field of Chentsov type on $\mathbf{R}^{d}$ associated with $\mu$. For $t \in \mathbf{R}^{a}$ let $S_{t}$ be the set defined by (1.2). For $T=\left(t_{1}, \cdots, t_{n}\right)$ $\in\left(\mathbf{R}^{d}\right)^{n}$ and $e=\left(e_{1}, \cdots, e_{n}\right) \in \mathscr{E}_{n}$, we write

$$
\begin{aligned}
& S_{t_{k}}^{e_{k}}= \begin{cases}S_{t_{k}} & \text { if } e_{k}=1 \\
S_{t_{k}}^{c} & \text { if } e_{k}=0\end{cases} \\
& \tilde{\boldsymbol{S}}_{t_{k}}^{e_{k}}= \begin{cases}S_{t_{k}} & \text { if } e_{k}=1 \\
\mathbf{R}_{+} \times \mathbf{R}^{d} & \text { if } e_{k}=0\end{cases} \\
& S(T, e)=\bigcap_{k=1}^{n} S_{t_{k}}^{e_{k}} \\
& \tilde{S}(T, e)=\bigcap_{k=1}^{n} \tilde{S}_{t_{k}}^{e_{k}} .
\end{aligned}
$$

$S(T, e)$ is an element (labelled with $e$ ) of the partition of the set $\bigcup_{k=1}^{n} S_{t_{k}}$ generated by $\left\{S_{t_{k}} ; k=1, \cdots, n\right\}$.

Definition 2.1. We say that $T=\left(t_{1}, \cdots, t_{n}\right) \in\left(\mathbf{R}^{d}\right)^{n}$ satisfies Condition (I) if there exists a label $e \in \mathscr{E}_{n}$ such that $S(T, e)=\varnothing$.

Remark. Suppose that $T=\left(t_{1}, \cdots, t_{n}\right)$ satisfies Condition (I). Then $T^{\prime}=\left(t_{1}, \cdots, t_{n}, t_{n+1}, \cdots, t_{m}\right)$ satisfies Condition (I) for any $t_{n+1}, \cdots, t_{m} \in \mathbf{R}^{d}$. In fact, suppose that $S(T, e)=\varnothing$ for $e=\left(e_{1}, \cdots, e_{n}\right) \in \mathscr{E}_{n}$. If we take a label $\tilde{e}=\left(\tilde{e}_{1}, \cdots, \tilde{e}_{m}\right)$ such that $\tilde{e}_{1}=e_{1}, \cdots, \tilde{e}_{n}=e_{n}$, then $S\left(T^{\prime}, \tilde{e}\right)=\varnothing$.

Lemma 2.2. Let $t_{1}, \cdots, t_{n}$ be different points in $\mathbf{R}^{d}$. If $T=\left(t_{1}, \cdots, t_{n}\right)$ 
satisfies Condition (I), then the distribution of $X_{T}=\left(X\left(t_{1}\right), \ldots, X\left(t_{n}\right)\right)$ is determined by the system of $(n-1)$-dimensional marginal distributions of $X_{T}$.

Proof. The characteristic function of $X_{T}$ is written, for $z=\left(z_{1}, \cdots, z_{n}\right)$ $\in \mathbf{R}^{n}$, as

$$
\begin{aligned}
\varphi_{T}(z) & =E \exp \left\{i \sum_{k=1}^{n} z_{k} Y\left(S_{t_{k}}\right)\right\} \\
& =E \exp \left\{i \sum_{k=1}^{n} z_{k} \sum_{\substack{e \in \varepsilon_{n} \\
e_{k}=1}} Y(S(T, e))\right\} \\
& =E \exp \left\{i \sum_{e \in \boldsymbol{\theta}_{n}}\left(\sum_{k=1}^{n} e_{k} z_{k}\right) Y(S(T, e))\right\} \\
& =\exp \left\{-\sum_{e \in \delta_{n}}\left|\sum_{k=1}^{n} e_{k} z_{k}\right|^{\alpha} \mu(S(T, e))\right\} . \\
& =\exp \left\{-\sum_{e \in \delta_{n}}|\xi(e) \cdot z|^{\alpha}(\# e)^{\alpha / 2} \mu(S(T, e))\right\},
\end{aligned}
$$

where $e=\left(e_{1}, \cdots, e_{n}\right)$, \#e= $\sum_{i=1}^{n} e_{i}$, and

$$
\xi(e)=\left(1 /(\# e)^{1 / 2}\right) e .
$$

On the other hand it is known that the characteristic function of an $n$-dimensional $S \alpha S$ distribution, $0<\alpha<2$, has the following unique canonical representation:

$$
\varphi(z)=\exp \left\{-c \int_{S^{n-1}}|\xi \cdot z|^{\alpha} \lambda(d \xi)\right\},
$$

where $c>0$ and $\lambda$ is a symmetric probability measure on the unit sphere $S^{n-1}$ [2]. Comparing the last expression of (2.5) to (2.7) and noticing that $\xi(e)$ of (2.6) belongs to $S^{n-1}$ for any $e$, we see that the last expression of (2.5) gives the canonical form of $\varphi_{T}(z)$. So, we see that $\varphi_{T}(z)$ is determined by the values of $\mu(S(T, e)), e \in \mathscr{E}_{n}$, and that, conversely, $\mu(S(T, e))$, $e \in \mathscr{E}_{n}$, are determined by $\varphi_{T}(z)$.

Since $\mu$ is a measure, the following consistency condition holds:

$$
\mu(\tilde{S}(T, e))=\sum_{e^{\prime} \in \delta_{n^{\prime}}(e)} \mu\left(S\left(T, e^{\prime}\right)\right) \quad \text { for each } e \in \mathscr{E}_{n},
$$

where, for $e=\left(e_{1}, \cdots, e_{n}\right) \in \mathscr{E}_{n}, \mathscr{E}_{n}^{\prime}(e)$ is the subset of $\mathscr{E}_{n}$ defined by

$$
\mathscr{E}_{n}^{\prime}(e)=\left\{e^{\prime}=\left(e_{1}^{\prime}, \cdots, e_{n}^{\prime}\right) ; e_{k}^{\prime} \geqq e_{k} \quad \text { for } k=1, \cdots, n\right\} .
$$

Since the number of the elements of $\mathscr{E}_{n}$ is $2^{n}-1$, (2.8) consists of $2^{n}-1$ equations. But one of them, that is, the case $e=(1, \cdots, 1)$, is a trivial 
equation. So we consider

$$
\sum_{e^{\prime} \in \mathscr{E}^{\prime}(e)} \mu\left(S\left(T, e^{\prime}\right)\right)=\mu(\tilde{S}(T, e)) \quad \text { for } e \in \mathscr{E}_{n} \backslash\{(1, \cdots, 1)\}
$$

as a system of $2^{n}-2$ simultaneous linear equations in which the unknowns are the values of $\mu\left(S(T, e)\right.$ ), $e \in \mathscr{E}_{n}$, (the number of them is $2^{n}-1$ ) and the given right-hand sides are the values of $\mu(\tilde{S}(T, e)), e \in \mathscr{E}_{n} \backslash\{(1, \cdots, 1)\}$. These right-hand sides are determined by the $(n-1)$-dimensional marginal distributions of $X_{T}$. We write the matrix representation of the system (2.10) as

$$
M_{n} \mathrm{x}=\mathbb{b},
$$

where $M_{n}$ is a $\left(2^{n}-2\right) \times\left(2^{n}-1\right)$-matrix, whose components are 0 or 1 . Let $M_{n}(k)$ be the $\left(2^{n}-2\right) \times\left(2^{n}-2\right)$-matrix obtained from $M_{n}$ by deleting the $k$-th column. Then we can prove that

$$
M_{n}(k) \text { is invertible for any } k=1, \cdots, 2^{n}-1 .
$$

Proof of (2.12) will be given at the end of this section. By the assumption that $T$ satisfies Condition (I), there exists a label $e$ such that $S(T, e)=\varnothing$. This implies $\mu(S(T, e))=0$ for the label $e$. So, the number of the unknowns is reduced to $2^{n}-2$. Suppose that the $\mu(S(T, e))$ corresponding to the label $e$ is the $k$-th component of the column vector of the unknowns. Then our system of simultaneous linear equations is equivalent to the system having $M_{n}(k)$ as its coefficient matrix. By virtue of (2.12) the system of equations has a unique solution. Thus, all the unknowns are determined.

Now we need to study the problem when $T$ satisfies Condition (I).

Let $T=\left(t_{1}, \cdots, t_{n}\right) \in\left(\mathbf{R}^{d}\right)^{n}$ and $e=\left(e_{1}, \cdots, e_{n}\right) \in \mathscr{E}_{n}$. The set $S(T, e)$ is partitioned into two disjoint subsets:

$$
S(T, e)=\left\{S(T, e) \cap C_{0}\right\} \cup\left\{S(T, e) \cap C_{0}^{c}\right\},
$$

where $C_{0}$ is the cone with vertex 0 .

LEMMA 2.3. We have

$$
\begin{aligned}
& S(T, e) \cap C_{0}=C\left(T, e^{*}\right) \cap C_{0}, \\
& S(T, e) \cap C_{0}^{c}=C(T, e) \cap C_{0}^{c},
\end{aligned}
$$

where $e^{*}$ is the complementary element of $e$.

Proof. We have 


$$
S(T, e) \cap C_{0}=\left(\bigcap_{i=1}^{n} S_{t_{i}}^{e_{i}}\right) \cap C_{0}=\bigcap_{i=1}^{n}\left(S_{t_{i}}^{e_{i}} \cap C_{0}\right) .
$$

If $e_{i}=1$, then

$$
S_{t_{i}}^{e_{i}} \cap C_{0}=S_{t_{i}} \cap C_{0}=\left(C_{t_{i}} \triangle C_{0}\right) \cap C_{0}=C_{t_{i}}^{c} \cap C_{0}=C_{t_{i}}^{e^{*}} \cap C_{0} .
$$

If $e_{i}=0$, then

$$
S_{t_{i}}^{e_{i}} \cap C_{0}=S_{t_{i}}^{c} \cap C_{0}=\left(C_{t_{i}} \triangle C_{0}\right)^{c} \cap C_{0}=C_{t_{i}} \cap C_{0}=C_{t_{i}}^{e_{i}^{*}} \cap C_{0} .
$$

Hence

$$
\bigcap_{i=1}^{n}\left(S_{t_{i}}^{e_{i}} \cap C_{0}\right)=\bigcap_{i=1}^{n}\left(C_{t_{i}}^{e^{*}} \cap C_{0}\right)=\left(\bigcap_{i=1}^{n} C_{t_{i}}^{e^{*}}\right) \cap C_{0}=C\left(T, e^{*}\right) \cap C_{0} .
$$

This proves (2.14). The relation (2.15) is obtained more easily.

Using Lemma 2.3 we can reduce Condition (I) to the following Condition (II).

Definition 2.4. We say that $T=\left(t_{1}, t_{2}, \cdots, t_{m}\right) \in\left(\mathbf{R}^{d}\right)^{m}$ satisfies Condition (II) if there exists a label $e \in \mathscr{E}_{m}$ such that both $C(T, e)=\varnothing$ and $C\left(T, e^{*}\right)=\varnothing$ hold.

Lemma 2.5. $T=\left(t_{1}, \cdots, t_{n}\right) \in\left(\mathbf{R}^{d}\right)^{n}$ satisfies Condition (I) if and only if $\tilde{T}=\left(0, t_{1}, \cdots, t_{n}\right) \in\left(\mathbf{R}^{d}\right)^{n+1}$ satisfies Condition (II).

Proof. (i) Suppose that $T=\left(t_{1}, \cdots, t_{n}\right)$ satisfies Condition (I). Let $e=\left(e_{1}, \cdots, e_{n}\right) \in \mathscr{E}_{n}$ be the label such that $S(T, e)=\varnothing$. It follows from (2.13) and Lemma 2.3 that $C\left(T, e^{*}\right) \cap C_{0}=\varnothing$ and $C(T, e) \cap C_{0}^{c}=\varnothing$. Put $\tilde{e}=\left(0, e_{1}, \cdots, e_{n}\right) \in \mathscr{E}_{n+1}$. Then

$$
\begin{aligned}
& C\left(T, e^{*}\right) \cap C_{0}=C\left(\tilde{T}, \tilde{e}^{*}\right), \\
& C(T, e) \cap C_{0}^{c}=C(\tilde{T}, \tilde{e}) .
\end{aligned}
$$

Hence $\tilde{T}$ satisfies Condition (II).

(ii) Suppose that $\tilde{T}=\left(0, t_{1}, \cdots, t_{n}\right)$ satisfies Condition (II). Let $\tilde{e}=$ $\left(e_{0}, e_{1}, \cdots, e_{n}\right) \in \mathscr{E}_{n+1}$ be the label such that $C(\tilde{T}, \tilde{e})=\varnothing$ and $C\left(\tilde{T}, \tilde{e}^{*}\right)=\varnothing$. Let $e=\left(e_{1}, \cdots, e_{n}\right)$. If $e_{0}=0$, then $e_{0}^{*}=1$ and

$$
\begin{aligned}
& \varnothing=C(\tilde{T}, \tilde{e})=C(T, e) \cap C_{0}^{c}, \\
& \varnothing=C\left(\tilde{T}, \tilde{e}^{*}\right)=C\left(T, e^{*}\right) \cap C_{0},
\end{aligned}
$$

which implies $S(T, e)=\varnothing$ by (2.13) and Lemma 2.3. If $e_{0}=1$, then $e_{0}^{*}=0$ and, taking account of $\left(e^{*}\right)^{*}=e$, we have $S\left(T, e^{*}\right)=\varnothing$. In either case, $T=\left(t_{1}, \cdots, t_{n}\right)$ satisfies Condition (I). 
Proof that Theorem 2 implies Theorem 1. We assume that Theorem 2 is valid. Let $n \geqq d+1$. Let $t_{1}, \cdots, t_{n}$ be distinct points in $\mathbf{R}^{d}$. Theorem 2 combined with Lemma 2.5 tells us that $T=\left(t_{1}, \cdots, t_{n}\right)$ satisfies Condition (I). Hence, by Lemma 2.2 the distribution of $X_{T}$ is determined by its $(n-1)$-dimensional marginal distributions. Further, if $n-1 \geqq d+2$, then the $(n-1)$-dimensional marginal distributions of $X_{T}$ are determined by their $(n-2)$-dimensional marginal distributions. Proceeding in this way we see that the distributions of $X_{T}$ is determined by its $(d+1)$ dimensional marginal distributions. Theorem 1 is proved.

Proof of (2.12). To write down the matrix $M_{n}$, we introduce a linear order among the element of $\mathscr{E}_{n}$. Let $n(e)=\sum_{i=1}^{n} 2^{i-1} e_{i}$ for $e=\left(e_{1}, \cdots, e_{n}\right)$. We define $e<e^{\prime}$ if $n(e)<n\left(e^{\prime}\right)$. This gives a linear order in $\mathscr{E}_{n}$. Thus the first element is $(1,0,0, \cdots, 0)$ and the last element is $(1, \cdots, 1)$. We have

$$
\begin{aligned}
M_{2}= & \left(\begin{array}{lll}
1 & 0 & 1 \\
0 & 1 & 1
\end{array}\right) \\
M_{3}= & \left(\begin{array}{lllllll}
1 & 0 & 1 & 0 & 1 & 0 & 1 \\
0 & 1 & 1 & 0 & 0 & 1 & 1 \\
0 & 0 & 1 & 0 & 0 & 0 & 1 \\
0 & 0 & 0 & 1 & 1 & 1 & 1 \\
0 & 0 & 0 & 0 & 1 & 0 & 1 \\
0 & 0 & 0 & 0 & 0 & 1 & 1
\end{array}\right) .
\end{aligned}
$$

Let $N_{n}=2^{n}-1=2 N_{n-1}+1$. Then $M_{n}$ is a $\left(N_{n}-1\right) \times N_{n}$-matrix. It is easy to see that

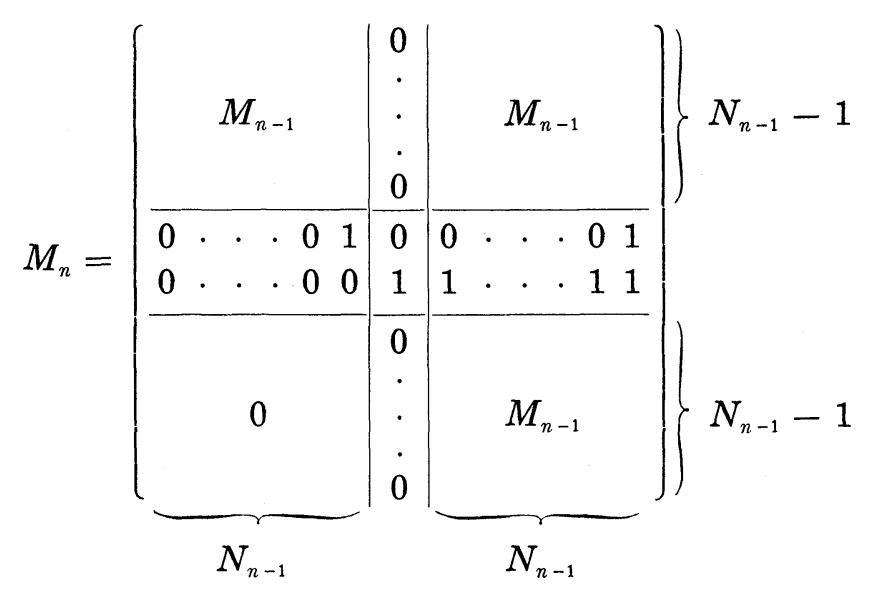


for $n \geqq 3$. Let $a_{j}$ be the $j$-th column vector and $a_{i j}$ be the $(i, j)$-component of $M_{n}$. If we delete the last column in $M_{n}$, then we get an upper triangular matrix $M_{n}\left(N_{n}\right)$ with diagonal elements 1 . Hence

$$
\mathrm{a}_{1}, \cdots, \mathrm{a}_{N_{n}-1}
$$

are linearly independent. Now we claim the following.

$$
\begin{aligned}
& \text { If } c_{1} \mathrm{a}_{1}+c_{2} \mathrm{a}_{2}+\cdots+c_{N_{n}} \mathrm{a}_{N_{n}}=0 \text { with } c_{k}=0 \\
& \text { for some } k \neq N_{n} \text {, then } c_{N_{n}}=0 .
\end{aligned}
$$

Suppose that (2.18) is true. Then we see that $M_{n}(k)$ is invertible for every $k$. Indeed, if $k=N_{n}$, then $M_{n}(k)$ is invertible by (2.17). If $k \neq N_{n}$, then (2.17) and (2.18) show that the column vectors of $M_{n}(k)$ are linearly independent.

It remains to prove the assertion (2.18). It suffices to show that the relation

$$
c_{1} \mathrm{a}_{1}+c_{2} \mathrm{a}_{2}+\cdots+c_{N_{n}} \mathrm{a}_{N_{n}}=0 \text { with } \quad c_{N_{n}}=1
$$

implies that

$$
c_{k} \neq 0 \text { for } k=1, \cdots, N_{n}-1 \text {. }
$$

Note that, by (2.17), all of $c_{1}, \cdots, c_{N_{n-1}}$ are determined uniquely by (2.19).

Denote the row vector

$$
\mathbb{C}_{n}=\left(c_{1}, c_{2}, \cdots, c_{N_{n}}\right) .
$$

Using the column vectors of $M_{n-1}$ in place of those of $M_{n}$, we get the row vector $\mathbb{C}_{n-1}$ in place of $\mathbb{C}_{n}$. Let

$$
\mathbb{C}_{n-1}=\left(\gamma_{1}, \gamma_{2}, \cdots, \gamma_{N_{n-1}}\right), \quad \gamma_{N_{n-1}}=1
$$

We write (2.19) componentwise:

$$
c_{1} a_{i 1}+c_{2} a_{i 2}+\cdots+c_{N_{n}} a_{i N_{n}}=0, i=1, \cdots, N_{n}-1, \text { and } c_{N_{n}}=1 .
$$

For $i=N_{n-1}+2, \cdots, N_{n}-1$, the relation between $M_{n}$ and $M_{n-1}$ in (2.16) shows that (2.21) implies

$$
\left(c_{N_{n-1}+2}, \cdots, c_{N_{n}}\right)=\left(\gamma_{1}, \cdots, \gamma_{N_{n-1}}\right) .
$$

For $i=N_{n-1}$, (2.21) reduces to $c_{N_{n-1}}+c_{N_{n}}=0$ by virtue of (2.16). Hence

$$
c_{N_{n-1}}=-1 \text {. }
$$


For $i=1, \cdots, N_{n-1}-1$, taking account of (2.16) and using (2.22) and (2.23), we have

$$
\left(c_{1}, c_{2}, \cdots, c_{N_{n-1}}\right)=\left(-\gamma_{1},-\gamma_{2}, \cdots,-\gamma_{N_{n-1}}\right) .
$$

It follows from (2.22) and (2.24) that

$$
\mathbb{C}_{n}=\left(-\mathbb{C}_{n-1}, c_{N_{n-1}+1}, \mathbb{C}_{n-1}\right) .
$$

We have

$$
\mathbb{C}_{2}=(-1,-1,1)
$$

explicitly from $M_{2}$. For $i=N_{n-1}+1,(2.21)$ reduces to

$$
c_{N_{n-1}+1}+c_{N_{n-1}+2}+\cdots+c_{N_{n}}=0
$$

by (2.16). Hence, noticing (2.22) and using (2.26) or (2.25) for $n-1$ in place of $n$, we get

$$
c_{N_{n-1}+1}= \begin{cases}1 & \text { for } n=3 \\ -\gamma_{N_{n-2}+1} & \text { for } n \geqq 4 .\end{cases}
$$

Now, from (2.25) and (2.27) we see that each component of $\mathbb{C}_{n}$ is 1 or -1 . This proves (2.20).

\section{§ 3. Proof of Theorem 2}

We prepare lemmas.

Lemma 3.1. Let $t_{i} \in \mathbf{R}^{d}, i=1, \cdots, n+m$. Let $A=\left\{t_{1}, \cdots, t_{n}\right\}$ and $B=\left\{t_{n+1}, \cdots, t_{n+m}\right\}$.

$$
\bigcap_{t_{i} \in A} C_{t_{i}} \subset \bigcup_{t_{j} \in B} C_{t_{j}}
$$

if and only if

$$
\max _{t_{i} \in A} d\left(t_{i}, x\right) \geqq \min _{t_{j} \in B} d\left(t_{j}, x\right) \quad \text { for any } x \in \mathbf{R}^{i} .
$$

We denote the relation (3.1) by $A \prec B$. This means

$$
\left(\bigcap_{t_{i} \in A} C_{t_{i}}\right) \cap\left(\bigcap_{t_{j} \in B} C_{t_{j}}^{c}\right)=\varnothing
$$

Proof. Suppose (3.1). Let $x \in \mathbf{R}^{d}$. Let $r=\max _{t_{i} \in A} d\left(x, t_{i}\right)$. Then $d\left(x, t_{i}\right) \leqq r$ for any $t_{i} \in A$, that is, $(r, x) \in \bigcap_{t_{i} \in A} C_{t_{i}}$. Hence $(r, x) \in \bigcup_{t_{j} \in B} C_{t_{j}}$. This means $d\left(x, t_{j}\right) \leqq r$ for some $t_{j} \in B$. Hence (3.2) holds. Conversely, assume (3.2). Let $(r, x) \in \bigcap_{t_{i} \in A} C_{t_{i}}$. That means $d\left(x, t_{i}\right) \leqq r$ for every $t_{i} \in A$. 
It follows from (3.2) that there exists $t_{j_{0}}$ such that $d\left(t_{j_{0}}, x\right) \leqq r$. So, $(r, x) \in C_{t_{j_{0}}}$.

LEMmA 3.2. Let $1 \leqq k \leqq d+1$. Let $t_{1}, \cdots, t_{k}$, and $t_{k+1}, \cdots, t_{d+2}$ in $\mathbf{R}^{d}$ be such that there is no hyperplane of co-dimension 1 containing $t_{1}, \cdots, t_{k}$, $t_{k+1}, \cdots, t_{d+1}$. Suppose that there exist positive constants $p_{1}, \cdots, p_{k}$ and $q_{k+1}, \cdots, q_{d+2}$ such that

$$
\begin{aligned}
& \sum_{i=1}^{k} p_{i}=\sum_{j=k+1}^{d+2} q_{j}=1, \\
& \sum_{i=1}^{k} p_{i} t_{i}=\sum_{j=k+1}^{d+2} q_{j} t_{j} .
\end{aligned}
$$

Then at least one of the following holds:

$$
\begin{aligned}
& \left.\bigcap_{i=1}^{k} C_{t_{i}} \subset \bigcup_{j=k+1}^{d+2} C_{t_{j}} \quad \text { (that is } A \prec B\right), \\
& \bigcup_{i=1}^{k} C_{t_{i}} \supset \bigcap_{j=k+1}^{d+2} C_{t_{j}} \quad(\text { that is } A>B),
\end{aligned}
$$

where $A=\left\{t_{1}, \cdots, t_{k}\right\}$ and $B=\left\{t_{k+1}, \cdots, t_{d+2}\right\}$.

Proof. Let $D$ be the $(d-1)$-dimensional sphere on which the points $t_{1}, \cdots, t_{k}, t_{k+1}, \cdots, t_{d+1}$ lie. Without loss of generality, we assume that the center of $D$ is $O=(0, \cdots, 0) \in \mathbf{R}^{d}$. Let $r$ be the radius of $D$. Suppose that $\left|t_{d+2}\right| \leqq r$. We will show that (3.5) holds. By Lemma 3.1 it is enough to show that, for any $x \in \mathbf{R}^{d}$,

$$
\max _{i=1, \cdots, k} d\left(t_{i}, x\right)-\min _{j=k+1, \cdots, d+2} d\left(t_{j}, x\right) \geqq 0 .
$$

Taking account of (3.3) and (3.4) we have

$$
\min _{i=1, \cdots, k}\left(t_{i}, x\right) \leqq \sum_{i=1}^{k} p_{i}\left(t_{i}, x\right)=\sum_{j=k+1}^{d+2} q_{j}\left(t_{j}, x\right) \leqq \max _{j \rightarrow k+1, \cdots, d+2}\left(t_{j}, x\right)
$$

where $(x, y)$ denotes inner product of $R^{t}$. Let $i_{0}$ and $j_{0}$ be the elements which attain the minimum and the maximum in (3.8), respectively. Then

$$
\left(t_{j_{0}}-t_{i_{0}}, x\right) \geqq 0 \text {. }
$$

On the other hand,

$$
\begin{aligned}
\max _{i=1, \cdots, k} & \left\{d\left(t_{i}, x\right)\right\}^{2}-\min _{j=k+1, \cdots, d+2}\left\{d\left(t_{j}, x\right)\right\}^{2} \\
& \geqq\left\{d\left(t_{i_{0}}, x\right)\right\}^{2}-\left\{d\left(t_{j_{0}}, x\right)\right\}^{2} \\
& =\left\{\left|t_{i_{0}}\right|^{2}+|x|^{2}-2\left(t_{i_{0}}, x\right)\right\}-\left\{\left|t_{j_{0}}\right|^{2}+|x|^{2}-2\left(t_{j_{0}}, x\right)\right\} \\
& =\left|t_{i_{0}}\right|^{2}-\left|t_{j_{0}}\right|^{2}+2\left(t_{j_{0}}-t_{i_{0}}, x\right) .
\end{aligned}
$$


(3.9) and the assumption $\left|t_{i_{0}}\right|=r \geqq\left|t_{j_{0}}\right|$ impliy that the last term of (3.10) is non-negative. So, (3.7) is proved.

Suppose that $\left|t_{d+2}\right| \geqq r$. Then we prove that

$$
\max _{j=k+1, \cdots, d+2} d\left(t_{j}, x\right)-\min _{i=1, \cdots, k} d\left(t_{i}, x\right) \geqq 0 \quad \text { for any } x \in R^{d},
$$

which implies (3.6) by Lemma 3.1. In fact, let

$$
\left(t_{i_{0}}, x\right)=\max _{i=1, \cdots, k}\left(t_{i}, x\right), \quad\left(t_{j_{0}}, x\right)=\min _{j=k+1, \ldots, d+2}\left(t,,_{j} x\right) .
$$

Then

$$
\begin{aligned}
& \max _{j=k+1, \cdots, d+2}\left\{d\left(t_{j}, x\right)\right\}^{2}-\min _{i=1, \cdots, k}\left\{d\left(t_{i}, x\right)\right\}^{2} \\
& \geqq\left\{d\left(t_{j_{0}}, x\right)\right\}^{2}-\left\{d\left(t_{i_{0}}, x\right)\right\}^{2} \\
& \quad=\left|t_{j_{0}}\right|^{2}-\left|t_{i_{0}}\right|^{2}+2\left(t_{i_{0}}-t_{j_{0}}, x\right) \geqq 0
\end{aligned}
$$

which is (3.11).

Lemma 3.3. Let $t_{1}, \cdots, t_{d+1} \in \mathbf{R}^{d}$. Suppose that no hyperplane of codimension 1 contains them and that any $d$ vectors out of $t_{1}, \cdots, t_{d+1}$ are linearly independent. Let $t_{d+2}=0$. Then the set $\left\{t_{1}, \cdots, t_{d+1}, t_{d+2}\right\}$ is uniquely partitioned into two disjoint sets $A, B$ such that $A \neq \varnothing, B \ni t_{d+2}$ and there exist positive constants $p_{i}$ 's and $q_{j}$ 's satisfying

$$
\begin{aligned}
& \sum_{t_{i} \in A} p_{i} t_{i}=\sum_{t_{j} \in B} q_{j} t_{j}, \\
& \sum_{t_{i} \in A} p_{i}=\sum_{t_{j} \in B} q_{j}=1 .
\end{aligned}
$$

Proof. Since $t_{1}, \cdots, t_{d+1}$ are linearly dependent, there exist constants $c_{1}, \cdots, c_{d+1}$ such that $\left(c_{1}, \cdots, c_{d+1}\right) \neq(0, \cdots, 0)$ and $\sum_{i=1}^{d+1} c_{i} t_{i}=0$. Notice that $c_{i} \neq 0$ for any $i$ by the assumption that any $d$ out of $t_{1}, \cdots, t_{d+1}$ are linearly independent. Moreover, $c_{1}, \cdots, c_{d+1}$ are unique up to constant multiple. We have $\sum_{i=1}^{d+1} c_{i} \neq 0$, because, if it is zero, then $\sum_{i=1}^{d} c_{i}\left(t_{i}-t_{d+1}\right)$ $=0$ and $t_{1}, \cdots, t_{d+1}$ are on a hyperplane of co-dimension 1 . So, we may assume that $\sum_{i=1}^{d+1} c_{i}>0$. Let $A=\left\{t_{i} ; c_{i}>0\right\}$ and $B=\left\{t_{i} ; c_{i}<0\right\} \cup\left\{t_{d+2}\right\}$. Let $p_{i}=c_{i}$ for $c_{i}>0, q_{j}=-c_{j}$ for $c_{j}<0$, and $q_{d+2}=\sum_{i=1}^{d+1} c_{i}$. Then $\sum_{t_{i} \in A} p_{i}-\sum_{t_{j} \in B} q_{j}=0$ and (3.12) holds. Multiplication of some constant yields (3.13). Uniqueness of $A$ and $B$ is obvious from this argument.

Corollary 3.4. Let $t_{i} \in \mathbf{R}^{d}, i=1, \cdots, d+2$. Assume that no $d+1$ points out of them are contained in a hyperplane of co-dimension 1 in $\mathbf{R}^{d}$. 
Then the set $\left\{t_{1}, \cdots, t_{d+2}\right\}$ is partitioned into two disjoint non-empty sets $A$ and $B$ such that, for some $p_{i}>0$ and $q_{j}>0$,

$$
\sum_{t_{i} \in A} p_{i} t_{i}=\sum_{t_{j} \in B} q_{j} t_{j}, \quad \sum_{t_{i} \in A} p_{i}=\sum_{t_{j} \in B} q_{j}=1 .
$$

The partition is unique up to the naming of $A$ and $B$.

Proof. Let $u_{i}=t_{i}-t_{d+2}$ and apply Lemma 3.3 to $u_{1}, \cdots, u_{d+2}$.

We call $A, B$ in Corollary 3.4 the natural partition of $\left\{t_{1}, \cdots, t_{d+2}\right\}$.

The corollary above is rephrased geometrically as follows. For a finite set $C=\left\{t_{1}, \cdots, t_{n}\right\} \subset \mathbf{R}^{d}$, denote by $\bar{C}$ the solid simplex having $C$ as the set of vertices, that is,

$$
\bar{C}=\left\{\sum_{i=1}^{n} p_{i} t_{i} ; \sum_{i=1}^{n} p_{i}=1, p_{i} \geqq 0, i=1, \cdots, n\right\} .
$$

Corollary 3.5. Let $t_{i}, i=1, \cdots, d+2$, be as in Corollary 3.4. Then there are two disjoint non-empty sets $A, B$ such that $A \cup B=\left\{t_{1}, \cdots, t_{d+2}\right\}$, $A \cap B=\varnothing$, and $\bar{A} \cap \bar{B} \neq \varnothing$. The sets $A, B$ are unique up to naming of $A$ and $B$. The set $\bar{A} \cap \bar{B}$ consists of only one point.

Combining Lemma 3.1 and Corollary 3.4, we get the following proposition.

Proposition 3.6. For any $T=\left(t_{1}, \cdots, t_{d+2}\right) \in\left(\mathbf{R}^{d}\right)^{d+2}$ such that no $d+1$ points out of $t_{1}, \cdots, t_{d+2}$ are contained in a hyperplane of co-dimension 1 in $\mathbf{R}^{d}$, there exists a label $e \in \mathscr{E}_{d+2}$ which satisfies $C(T, e)=\varnothing$.

Now we deal with a set of $d+3$ points in $\mathbf{R}^{d}$ in order to discuss Condition (II). Consider a set $\Gamma=\left\{t_{1}, \cdots, t_{d+3}\right\}$ in $\mathbf{R}^{d}$. Assume that $\Gamma$ is non-degenerate in the sense that

$$
\text { no } d+1 \text { points out of } t_{1}, \cdots, t_{d+3} \text { are contained in }
$$

a hyperplane of co-dimension 1 in $\mathbf{R}^{d}$.

For each $i$, apply Corollary 3.4 to $\Gamma \backslash\left\{t_{i}\right\}$ and let

$$
\Gamma \backslash\left\{t_{i}\right\}=A_{i} \cup B_{i}
$$

be the natural partition of $\Gamma \backslash\left\{t_{i}\right\}$. By Lemma 3.2, at least one of $A_{i} \prec B_{i}$ and $A_{i} \succ B_{i}$ holds.

Let $i \neq j$. We say that $t_{i}$ and $t_{j}$ link together if the restrictions to $\Gamma \backslash\left\{t_{i}, t_{j}\right\}$ of the natural partitions of $\Gamma \backslash\left\{t_{i}\right\}$ and $\Gamma \backslash\left\{t_{j}\right\}$ coincide. 
Lemma 3.7. Let $i \neq j$ and suppose that $t_{i}$ and $t_{j}$ link together. Let $A_{i}, B_{i}$ and $A_{j}, B_{j}$ be the natural partitions of $\Gamma \backslash\left\{t_{i}\right\}$ and $\Gamma \backslash\left\{t_{j}\right\}$, respectively. If

$$
A_{i} \prec B_{i}, \quad A_{j} \succ-B_{j}, \quad A_{i} \cap A_{j} \neq \varnothing,
$$

then $T=\left(t_{1}, \cdots, t_{d+3}\right)$ satisfies Condition (II).

Proof. Without loss of generality we assume $i=1, j=2$. Keeping $A_{1} \cap A_{2} \neq \varnothing$ in mind, we can find $A$ and $B$ satisfying $A \cup B=\Gamma \backslash\left\{t_{1}, t_{2}\right\}$ and $A \cap B=\varnothing$ such that one of the following four conditions holds:

(a) $A_{1}=A \cup\left\{t_{2}\right\}, \quad B_{1}=B, \quad A_{2}=A \cup\left\{t_{1}\right\}, \quad B_{2}=B$;

(b) $A_{1}=A \cup\left\{t_{2}\right\}, \quad B_{1}=B, \quad A_{2}=A, \quad B_{2}=B \cup\left\{t_{1}\right\}$;

(c) $A_{1}=A, \quad B_{1}=B \cup\left\{t_{2}\right\}, \quad A_{2}=A, \quad B_{2}=B \cup\left\{t_{1}\right\}$;

(d) $A_{1}=A, \quad B_{1}=B \cup\left\{t_{2}\right\}, \quad A_{2}=A \cup\left\{t_{1}\right\}, \quad B_{2}=B$.

We may assume that $A=\{3, \cdots, k\}$ and $B=\{k+1, \cdots, d+3\}$ where $3 \leqq k \leqq d+3(B=\varnothing$ if $k=d+3)$.

Case (a). We have

$$
\begin{aligned}
& C(T, e)=\varnothing \quad \text { with } \quad e=(e_{1}, \underbrace{1,1, \cdots, 1}_{k-1}, \underbrace{0, \cdots, 0}_{d+3-k}), \\
& C\left(T, e^{\prime}\right)=\varnothing \quad \text { with } \quad e=(0, e_{2}^{\prime}, \underbrace{0, \cdots, 0}_{k-2}, \underbrace{1, \cdots, 1}_{d+3-k}),
\end{aligned}
$$

whatever $e_{1}$ and $e_{2}^{\prime}$ are. Letting $e_{1}=1$ and $e_{2}^{\prime}=0$, we get a complementary pair $e, e^{\prime}$. Hence $T$ satisfies Condition (II).

Case (b). We have

$$
\begin{aligned}
& C(T, e)=\varnothing \quad \text { with } \quad e=(e_{1}, \underbrace{1,1, \cdots, 1}_{k-1}, \underbrace{0, \cdots, 0}) \\
& C\left(T, e^{\prime}\right)=\varnothing \quad \text { with } \quad e^{\prime}=(1, e_{2}^{\prime}, \underbrace{0, \cdots, 0}_{k-2}, \underbrace{1, \cdots, 1}_{d+3-k}),
\end{aligned}
$$

whatever $e_{1}$ and $e_{2}^{\prime}$ are. Letting $e_{1}=0$ and $e_{2}^{\prime}=0$, we obtain a complementary pair.

Cases (c) and (d) are treated similarly to (a) and (b), respectively.

Remark. Another sufficient condition for $T$ to satisfy Condition (II) is that there exists $i$ such that $A_{i} \prec B_{i}$ and $A_{i}>B_{i}$. But we will not use this condition.

We see easily that, to prove Theorem 2 , it is enough to prove it for $m=d+3$. In order to prove it for $m=d+3$ under the condition that 
$\left\{t_{1}, \cdots, t_{d+3}\right\}$ are non-degenerate in the sense of (3.15), we will show the existence of $i$ and $j$ which satisfy the condition of Lemma 3.7. Applying Corollary 3.4 to $\Gamma \backslash\left\{t_{1}\right\}$ and $\Gamma \backslash\left\{t_{2}\right\}$, we have

$$
\sum_{k=1}^{d+3} c_{1 k} t_{k}=0 \quad \text { with } \quad c_{11}=0, \quad \sum_{k=1}^{d+3} c_{1 k}=0, \quad c_{1 k} \neq 0(k \neq 1),
$$

and

$$
\sum_{k=1}^{d+3} c_{2 k} t_{k}=0 \quad \text { with } \quad c_{22}=0, \quad \sum_{k=1}^{d+3} c_{2 k}=0, \quad c_{2 k} \neq 0(k \neq 2) .
$$

The representations are unique up to constant multiplication. We assume $c_{12}>0$ and $c_{21}>0$. We set, for $i \geqq 3$,

$$
\left\{\begin{array}{l}
\lambda_{1}=c_{2 i} / c_{1 i} \\
c_{i k}=c_{2 k}-\lambda_{i} c_{1 k} .
\end{array}\right.
$$

Then we get the relations for $\Gamma \backslash\{i\}, i=3, \cdots, d+3$, that

$$
\sum_{k=1}^{d+3} c_{i k} t_{k}=0 \quad \text { with } \quad c_{i i}=0, \quad \sum_{k=1}^{d+3} c_{i k}=0 .
$$

Obviously we have, for $i \geqq 3$,

$$
\left\{\begin{array}{l}
c_{i 1}=c_{21}>0 \\
c_{i 2}=-\lambda_{i} c_{12} \\
c_{i k}=c_{1 k}\left(\lambda_{k}-\lambda_{i}\right)=c_{2 k}\left(1-\lambda_{i} / \lambda_{k}\right), \quad \text { for } k \geqq 3 .
\end{array}\right.
$$

Moreover we see that $\lambda_{i}, i=3, \cdots, d+3$, are distinct and $c_{i k} \neq 0$ for $k \neq i$, because, if otherwise, some $d+1$ points in $\Gamma$ are contained in a hyperplane of co-dimension 1 . Without loss of generality we assume $\lambda_{i}<\lambda_{i+1}$ for $i=3, \cdots, d+3$. Let

$$
L_{-}=\left\{i \geqq 3 ; \lambda_{i}<0\right\}, \quad L_{+}=\left\{j \geqq 3 ; \lambda_{j}>0\right\} .
$$

We see that $c_{i 2}>0$ for $i \in L_{-}$and $c_{j 2}<0$ for $j \in L_{+}$. Using the relations in (3.22) and noticing that the natural partition of $\Gamma \backslash\{i\}$ is made according to the signs of $c_{i k}$, we get

LEMMA 3.8. If both $i$ and $i+1$ belong to $L_{-}$, then $t_{i}$ and $t_{i+1}$ link together. If both $j$ and $j+1$ belong to $L_{+}$, then $t_{j}$ and $t_{j+1}$ link together.

Now we assume that

$$
L_{-} \neq \varnothing \quad \text { and } \quad L_{+} \neq \varnothing
$$


The case without this assumption will be treated later. Let

$$
L_{-}=\{3,4, \cdots, \gamma\}, \quad L_{+}=\{\gamma+1, \cdots, d+3\} .
$$

Then we get the following lemma.

LemMa 3.9. The following pairs link together:

(1) $t_{1}$ and $t_{3}$;

(2) $t_{1}$ and $t_{d+3}$;

(3) $t_{2}$ and $t_{r}$;

(4) $t_{2}$ and $t_{\gamma+1}$.

Proof. Again use (3.22) and the fact that the natural partition of $\Gamma \backslash\left\{t_{i}\right\}$ is decided by the signs of $c_{i k}, k \neq i$.

It follows from Lemma 3.7 that, if $L_{-}$or $L_{+}$contains adjacent elements $i, j$ satisfying (3.17), then $T=\left(t_{1}, \cdots, t_{d+3}\right)$ satisfies Condition (II). So, let us consider the situation that neither $L_{-}$nor $L_{+}$contains adjacent elements satisfying (3.17). In the naming of $A_{i}, B_{i}$ in the natural partition (3.16) of $\Gamma \backslash\left\{t_{i}\right\}$, we make $A_{i} \ni t_{1}$ for $i=2,3, \cdots, d+3$, and $A_{1} \ni t_{2}$. Recalling that the natural partitions are made by the signs of $c_{i k}$, we see that $t_{2} \in A_{i}$ for $i \in L_{-}$and that $t_{2} \in B_{i}$ for $i \in L_{+}$. We note that $A_{i-1} \cap A_{i} \neq \varnothing$ for $i \in L_{-} \cup L_{+}$. Hence Lemma 3.7 yields that we have one of the following situations:

(1) $A_{i} \prec B_{i}$ for $i \in L_{-} \cup L_{+}$;

(2) $A_{i} \prec B_{i}$ for $i \in L_{-}$and $A_{j} \succ B_{j}$ for $j \in L_{+}$;

(3) $A_{i} \succ B_{i}$ for $i \in L_{-} \cup L_{+}$;

(4) $A_{i} \succ B_{i}$ for $i \in L_{-}$and $A_{j} \prec B_{j}$ for $j \in L_{+}$.

We will prove that in each case at least one of pairs (1), (2), (3), (4) of Lemma 3.9 satisfies the condition of Lemma 3.7.

Case (1). If $A_{1} \prec B_{1}$, then $t_{1}$ and $t_{d+3}$ satisfy the condition of Lemma 3.7, because $A_{d+3} \prec B_{d+3}$ and $A_{1} \cap B_{d+3} \ni t_{2}$. If $A_{1}>B_{1}$, then $t_{1}$ and $t_{3}$ satisfy (3.17), since $A_{3} \prec B_{3}$ and $A_{1} \cap A_{3} \ni t_{2}$.

Case (2). If $A_{2} \prec B_{2}$, then $t_{2}$ and $t_{\gamma+1}$ satisfy (3.17), since $A_{\gamma+1} \succ B_{\gamma+1}$ and $A_{2} \cap A_{r+1} \ni t_{1}$. If $A_{2}>B_{2}$, then $t_{2}$ and $t_{r}$ satisfy (3.17), because $A_{r} \prec B_{r}$ and $A_{2} \cap A_{r} \ni t_{1}$.

Case (3). Similarly to case (1), the pair $t_{1}, t_{3}$ or the pair $t_{1}, t_{d+3}$ satisfies the condition of Lemma 3.7 . 
Case (4). Similar to case (2). The pair $t_{2}, t_{\gamma}$ or the pair $t_{2}, t_{r+1}$ satisfies (3.17).

Thus, under the assumption (3.24), $T=\left(t_{1}, \cdots, t_{d+3}\right)$ satisfies Condition (II).

Let us consider the case where $L_{-}$or $L_{+}$is empty.

LEMMA 3.10. If $L_{-}=\varnothing$, then each of the following pairs links together:

$$
t_{1}, t_{2} ; t_{1}, t_{d+3} ; t_{2}, t_{3} .
$$

If $L_{+}=\varnothing$, then each of the following pairs links together.

$$
t_{1}, t_{2} ; t_{1}, t_{3} ; t_{2}, t_{d+3} .
$$

Proof. Suppose that $L_{-}=\varnothing$. Let

$$
A=\left\{i \geqq 3 ; c_{1 i}>0, c_{2 i}>0\right\}, \quad B=\left\{i \geqq 3 ; c_{1 i}<0, c_{2 i}<0\right\} .
$$

Then $A \cup B=\{3, \cdots, d+3\}$, and hence $t_{1}$ and $t_{2}$ link together. If $L_{+}=\varnothing$, then letting

$$
A=\left\{i \geqq 3 ; c_{1 i}>0, c_{2 i}<0\right\}, \quad B=\left\{i \geqq 3 ; c_{1 i}<0, c_{2 i}>0\right\},
$$

we see that $A \cup B=\{3, \cdots, d+3\}$ and that $t_{1}$ and $t_{2}$ link together. The other assertions are proved in the same way by use of (3.22).

As before we make the naming of $A_{i}, B_{i}$ in the natural partition (3.16) in such a way that $A_{i} \ni t_{1}$ for $i=2,3, \cdots, d+3$, and $A_{1} \ni t_{2}$. We have $t_{2} \in A_{i}$ for $i \in L_{-}$and $t_{2} \in B_{i}$ for $i \in L_{+}$.

Suppose that $L_{-}=\varnothing$. If $L_{+}$contains adjacent elements $i, j$ satisfying (3.17), then, by Lemmas 3.7 and 3.8, $T=\left(t_{1}, \cdots, t_{d+3}\right)$ satisfies Condition (II). So, suppose that $L_{+}$does not contain adjacent elements satisfying (3.17). Then we have one of the following:

$$
\text { (1) } A_{i} \prec B_{i} \text { for } i \geqq 3, \quad \text { (2) } A_{i} \succ B_{i} \text { for } i \geqq 3 \text {. }
$$

Case (1). If $A_{1} \prec B_{1}$, then $t_{1}, t_{d+3}$ satisfy condition of Lemma 3.7 since $A_{d+3} \prec B_{d+3}$ and $A_{1} \cap B_{d+3} \ni t_{2}$. If $A_{2} \succ B_{2}$, then $t_{2}, t_{3}$ satisfy (3.15), since $A_{3} \prec B_{3}$ and $A_{2} \cap A_{3} \ni t_{1}$. In the remaining case, suppose that $A_{1} \succ B_{1}$ and $A_{2} \prec B_{2}$. If $c_{1 k}>0$ for some $k \geqq 3$, then $c_{2 k}>0$ and $A_{1} \cap A_{2} \ni t_{k}$. If $c_{1 k}<0$ for some $k \geqq 3$, then $c_{2 k}<0$ and $B_{1} \cap B_{2} \ni t_{k}$. So, $t_{1}, t_{2}$ satisfy the condition of Lemma 3.7. We made use of Lemma 1.10.

Case (2). If $A_{1}>B_{1}$, then $t_{1}, t_{d+3}$ satisfy the condition of Lemma 3.7, 
since $A_{d+3}>B_{d+3}$ and $A_{1} \cap B_{d+3} \ni t_{2}$. If $A_{2} \prec B_{2}$, then $t_{2}, t_{3}$ satisfy the condition, because $A_{3}>B_{3}$ and $A_{2} \cap A_{3} \ni t_{1}$. If $A_{1} \prec B_{1}$ and $A_{2}>B_{2}$, then $t_{1}, t_{2}$ satisfy the condition by same reason as case (1).

Suppose that $L_{+}=\varnothing$. Then we can make similar discussion. Namely, suppose that $L_{-}$does not contain adjacent elements satisfying (3.15). Then (1) or (2) holds. In either case we can find the following pair satisfying the condition of Lemma 3.7.

Case (1). If $A_{1}>B_{1}$, then $t_{1}, t_{3}$. If $A_{2}>B_{2}$, then $t_{2}, t_{d+3}$. If $A_{1} \prec B_{1}$ and $A_{2} \prec B_{2}$, then $t_{1}, t_{2}$.

Case (2). If $A_{1} \prec B_{1}$, then $t_{1}, t_{3}$. If $A_{2} \prec B_{2}$, then $t_{2}, t_{d+3}$. If $A_{1} \succ B_{1}$ and $A_{2} \succ B_{2}$, then $t_{1}, t_{2}$.

Therefore, in the case that $L_{-}$or $L_{+}$is empty, $T=\left(t_{1}, \cdots, t_{d+3}\right)$ satisfies Condition (II). This finishes proof of Theorem 2 for $m=d+3$ under the assumption that $t_{1}, \cdots, t_{d+3}$ are non-degenerate in the sense of (3.15).

If $d+1$ points are on a hyperplane of co-dimension 1 and no $d+2$ points are on a hyperplane of co-dimension 1 , then we can apply Lemma 3.2 again and similar argument can be made. If $d+2$ points are on a hyperplane of co-dimension 1, then, taking account of the remark to Definition 2.1, we see that the situation is reduced to $(d-1)$-dimensional case.

\section{$\S 4$. Concluding remarks}

In order to construct an example mentioned in Remark 1 of $\S 1$, we prepare a lemma.

LEMMA 4.1. Let $T=\left(t_{1}, \cdots, t_{d+2}\right) \in\left(\mathbf{R}^{d}\right)^{d+2}$, where $t_{1}, \cdots, t_{d+2}$ are distinct and no $d+1$ points of them are on a hyperplane of codimension 1 . Let $D$ be the $(d-1)$-dimensional sphere on which the points $t_{1}, \cdots, t_{d+1}$ lie. Assume that $t_{d+2}$ is situated inside of $D$ and, moreover, that $\bar{A} \cap \bar{B} \neq \varnothing$ for $A=\left\{t_{d+1}, t_{d+2}\right\}$ and $B=\left\{t_{1}, \cdots, t_{d}\right\}$, using the notation introduced before Corollary 3.5. Then there is no label $e$ of size $d+2$ such that $C(T, e)=$ $C\left(T, e^{*}\right)=\varnothing$.

Proof. For $e=\left(e_{1}, \cdots, e_{d+2}\right) \in \mathscr{E}_{d+2}$, let $A_{e}=\left\{t_{i} ; e_{i}=1\right\}$ and $B_{e}=\left\{t_{i} ; e_{i}=0\right\}$. In order to prove our assertion, it is enough to consider only $e$ such that $A_{e} \ni t_{d+2}$. We separate our discussion into three cases.

(a) $A_{e}$ and $B_{e}$ give the natural partition of $\left\{t_{1}, \cdots, t_{d+2}\right\}$. 
(b) Either $A_{e}$ or $B_{e}$ is a one point set.

(c) The remaining case.

Case (a). We have $A_{e}=A$ and $B_{e}=B$ by the assumption. From the proof of Lemma 3.2 we see that $A \succ B$. We do not have $A \prec B$. In fact, we can find a $(d-1)$-dimensional sphere $D^{\prime}$ such that $D^{\prime} \supset B$ and that the points $t_{d+1}, t_{d+2}$ and are inside of $D^{\prime}$. Let $x_{0}$ be the center of $D^{\prime}$. Then

$$
\max _{t_{i} \in A} d\left(t_{i}, x_{0}\right)<\min _{t_{j} \in B} d\left(t_{j}, x_{0}\right) .
$$

It follows from Lemma 3.1 that $A \prec B$ does not hold. Hence $C(T, e) \neq \varnothing$.

Case (b). If $\mathrm{A}_{e}$ consists of only one point $t_{i}$, then $C(T, e)$ contains a point $\left(\varepsilon, t_{i}\right)$ for sufficiently small $\varepsilon>0$. If $B_{e}$ consists of only one point, then $C\left(T, e^{*}\right) \neq \varnothing$.

Case (c). The sets $A_{e}, B_{e}$ do not give the natural partition of $\left\{t_{1}, \cdots, t_{d+2}\right\}$. So we have $\bar{A}_{e} \cap \bar{B}_{e}=\varnothing$ by the uniqueness of the natural partition. We can find a $(d-1)$-dimensional sphere $D^{\prime}$ such that $D^{\prime} \supset B_{e}$ and all the points of $A_{e}$ are inside of $D^{\prime}$. Then $C(T, e) \neq \varnothing$, since

$$
\max _{t_{i} \in A_{e}} d\left(t_{i}, x_{0}\right)<\min _{t_{j} \in B_{e}} d\left(t_{j}, x_{0}\right)
$$

for the center $x_{0}$ of $D^{\prime}$.

Example 4.2. Let $T_{0}=\left(t_{1}, \cdots, t_{d+1}\right) \in\left(\mathbf{R}^{d}\right)^{d+1}$ and $t_{d+2}=0$. We choose and fix $T_{0}$ in such a way that $T=\left(t_{1}, \cdots, t_{d+1}, t_{d+2}\right)$ satisfies the assumption in Lemma 4.1. It follows from Lemmas 2.5 and 4.1 that $S(T, e) \neq \varnothing$ for every $e \in \mathscr{E}_{d+1}$. Let $\mu$ be a measure on $E=\mathbf{R}_{+} \times \mathbf{R}^{d}$ satisfying (1.3) such that $\mu\left(S\left(T_{0}, e\right)\right)>0$ for every $e \in \mathscr{E}_{d+1}$. Let us define $\tilde{\mu}$ in the following way. We make $\tilde{\mu}=\mu$ on $E \backslash \bigcup_{i=1}^{d+1} S_{t_{i}}$. First notice that $\mu$ satisfies the consistency condition (2.11) for $n=d+1$. Using the notations in the proof of Lemma 2.2 , let $A$ be the matrix $M_{d+1}\left(2^{d+1}-1\right)$ and $b$ be the vector in (2.11). Let $\mathbb{C}$ be the $\left(2^{d+1}-2\right)$-vector every component of which is $\mu\left(\bigcap_{i=1}^{d+1} S_{t_{i}}\right)$. Choose $\varepsilon \neq 0$ such that every component of the solution $\mathrm{x}$ of

$$
A \mathrm{x}=\mathbb{b}-(1+\varepsilon) \mathbb{C}
$$

is positive. It suffices to make $|\varepsilon|$ small enough. Now let

$$
\tilde{\mu}\left(\bigcap_{i=1}^{d+1} S_{t_{i}}\right)=(1+\varepsilon) \mu\left(\bigcap_{i=1}^{d+1} S_{t_{i}}\right)
$$


and let $\tilde{\mu}\left(S\left(T_{0}, e\right)\right)$ for $e \in \mathscr{E}_{d+1} \backslash\{(1, \cdots, 1)\}$ be given by the solution $\mathbf{x}$. There exists a measure $\tilde{\mu}$ with these $\tilde{\mu}\left(S\left(T_{0}, e\right)\right), e \in \mathscr{E}_{d+1}$. We have $\tilde{\mu}\left(S_{t}\right)$ $<\infty$ for all $t \in \mathbf{R}^{d}$. Let $\{X(t)\}$ and $\{\tilde{X}(t)\}$ be the Chentsov type $S \alpha S$ random fields associated with $\mu$ and $\tilde{\mu}$, respectively. From the construction

$$
\tilde{\mu}\left(\tilde{S}\left(T_{0}, e\right)\right)=\mu\left(\tilde{S}\left(T_{0}, e\right)\right) \quad \text { for all } e \in \mathscr{E}_{d+1} \backslash\{(1, \cdots, 1)\} .
$$

It follows that $\left(X\left(t_{1}\right), \cdots, X\left(t_{d+1}\right)\right)$ and $\left(\tilde{X}\left(t_{1}\right), \cdots, \tilde{X}\left(t_{d+1}\right)\right)$ have different distributions but they have common $d$-dimensional marginal distributions.

ExAmple 4.3. An interesting problem is whether there are two measures $\mu$ and $\tilde{\mu}$ satisfying (1.3) such that the Chentsov type $S \alpha S$ random fields $\{X(t)\}$ and $\{\tilde{X}(t)\}$ on $\mathbf{R}^{d}$ associated with $\mu$ and $\tilde{\mu}$, respectively, have identical $d$-dimensional distributions but different $(d+1)$-dimensional distributions. We do not know the answer to this problem for general $d$ yet. But, in case $d=1$, we can construct such measures.

Let $E=\mathbf{R}_{+} \times \mathbf{R}^{1}$. Let $\mu$ be such that $\mu\left(S_{t}\right)=\mu\left(S_{-t}\right)<\infty$ and $\mu\left(S_{t}\right)$ is a continuous increasing function of $t>0$. Suppose, further, that $\mu$ is mutually absolutely continuous with the Lebesgue measure. Let $\tilde{\mu}$ be a measure concentrated on $\mathbf{R}_{+} \times\{0\}$ such that

$$
\tilde{\mu}\left(S_{t}\right)=\tilde{\mu}\left(S_{t} \cap\left(\mathbf{R}_{+} \times\{0\}\right)\right)=\mu\left(S_{t}\right) .
$$

Then $\{X(t)\}$ and $\{\tilde{X}(t)\}$ have common 1-dimensional distributions. Let $0<t_{1}<t_{2}$. Then $\mu\left(S_{t_{1}} \cap S_{t_{2}}^{c}\right)>0$ but $\tilde{\mu}\left(S_{t_{1}} \cap S_{t_{2}}^{c}\right)=0$, which implies that $\left(X\left(t_{1}\right), X\left(t_{2}\right)\right)$ and $\left(\tilde{X}\left(t_{1}\right), \tilde{X}\left(t_{2}\right)\right)$ have different distributions.

Our technique in this paper works in finding determinism of random fields on $\mathbf{R}^{d}$ of a similar sort.

THEOREM 4.4. Let $\mu$ be a measure on $\mathbf{R}_{+} \times \mathbf{R}^{d}$ satisfying $\mu\left(C_{t}\right)<\infty$ for every $t \in \mathbf{R}^{d}$ and let $Y(\cdot)$ be the $S \alpha S$ random measure associated with $\mu$. Let

$$
X(t)=Y\left(C_{t}\right) \quad \text { for } t \in \mathbf{R}^{a} .
$$

Then, for any $n>d$, any n-dimensional distribution of $\{X(t)\}$ is determined by its d-dimensional marginal distributions.

Proof. The non-degenerate case is dealt with Proposition 3.6 and Lemma 2.2. The degenerate case is obvious.

Finally we remark that, if $\mu$ is invariant under translation in $\mathbf{R}^{d}$, 
then $\left\{X(t) ; t \in \mathbf{R}^{d}\right\}$ in Theorem 4.4 is a homogeneous random field constructed geometrically.

Acknowledgment. The author expresses her sincere thanks to Professor T. Hida not only for his important comments to this paper, but also for his warm encouragement for many years. She is thankful to the referee for careful reading and valuable advice.

\section{REFERENCES}

[1] Chentsov, N. N., Lévy's Brownian motion of several parameters and generalized white noise, Theory Probab. Appl., 2 (1957), 265-266.

[2] Lévy, P., Théorie de l'addition des variables aléatoires, Gauthier-villars, Paris, (1937).

[ 3 ] — - Processus stochastiques et mouvement brownien, Gauthier-Villers, Paris. (1965, $2^{\text {ème }}$ edition)

[4] Sato, Y., Joint distributions of some self-similar stable processes, preprint (1989).

[5] - Structure of Lévy measures of stable random fields of Chentsov type, preprint (1990).

[ 6 ] Sato, Y. and Takenaka, S., On stable processes of Chentsov type, preprint (1990).

[7] Takenaka, S., Integral-geometric constructions of self-similar stable processes, Nagoya Math. J., 123 (1991),

Department of General Education

Aichi Institute of Technology

Yakusa-cho, Toyota 470-03

Japan 